\title{
Volksgesetzgebung in Brandenburg - Ein Bundesland auf dem Weg in eine andere Republik? - Eine Zwischenbilanz nach 10 Jahren
}

\author{
Markus Vette
}

Mit der Verabschiedung der Landesverfassung von Brandenburg durch den Volksentscheid am 14.06.1992 und Inkrafttreten am 20.08.1992 ging ein z.T. erbitterter Streit um das Verhältnis dieser Landesverfassung zum Grundgesetz zu Ende, in dessen Verlauf die Brandenburger Landesverfassung als Weg in eine andere Republik [1] bezeichnet wurde. Zu den Besonderheiten der Landesverfassung Brandenburgs gehört u. a., dass vergleichsweise geringe Quoren für die in der Verfassung verankerten Volksgesetzgebungsschritte [2] festgelegt wurden. Es wurde die Befürchtung in den Raum gestellt, Volksgesetzgebungsschritte würden unter diesen Bedingungen zu einer Ersetzung der repräsentativen parlamentarischen Demokratie durch Formen direkter Demokratie führen.

Hat sich die seinerzeit in der Verfassungsdebatte prophezeite inflationäre Benutzung dieses Gesetzgebungsweges - vor allem durch kleine aktive Minderheiten bewahrheitet?

Zur Mitte der dritten Wahlperiode des Landesparlaments, d. h. 10 Jahre nach der Bildung des Bundeslandes, soll eine Zwischenbilanz gezogen werden.

\section{Erste Wahlperiode 1990-1994}

In der ersten Wahlperiode gab es sieben Initiativen, die dem Landtagspräsidenten i. S. einer Volksinitiative übergeben wurden. In zwei Fällen war das Mindestquorum nicht erreicht, sechs der sieben Initiativen betrafen die Kreisgebietsreform in den Jahren 1992 und 1993. Während lokalen bzw. regionalen Interessen im Zuge der Kreisgebietsreform schlussendlich punktuell entsprochen wurde, bleiben die Volksinitiativen zur grundsätzlichen Übertragung der Kreisgebietsreform „an das Volk“ bzw. der Kreisstadtentscheidung grundsätzlich durch den Kreistag ohne Erfolg. Bemerkenswert ist, dass diese Reform in Brandenburg zügig und zugunsten des führenden Regierungspartners verlaufen ist, während Gebietsreformen in den westlichen Bundesländern häufig längere Zeiträume in Anspruch nahmen und zu einer tendenziellen Schwächung der die Reform vorantreibenden Regierung fuihrten.

Die siebente in der ersten Wahlperiode begonnene Volksinitiative richtete sich gegen den vorgesehenen Havelausbau, das Verkehrsprojekt 17 „Deutsche Einheit". Nahezu die Hälfte der dafür 1994 von den Initiatoren gesammelten 36.000 Unterschriften wurden als nicht zulässig beanstandet, sodass das Quorum von 20.000 Unterschriften nicht erreicht wurde.

\section{Zweite Wahlperiode 1994-1999}

Das Themenspektrum der Volksinitiativen in der zweiten Wahlperiode war im Vergleich zur ersten Wahlperiode dagegen deutlich breiter.

Die o. g. im Juli 1994 nicht erfolgreiche Volksinitiative gegen den Havelausbau legte im August 1995 erneut Unterschriftslisten vor. Diesmal wurde das Quorum sicher erreicht. Der Landtag lehnte die Volksinitiative am 13.12.1995 ab. Vom April bis August 1996 lief daraufhin der zweite Schritt der Brandenburger Volksgesetzgebung, das Volksbegehren, und erbrachte 58.306 gültige Eintragungen. Das Quorum von 80.000 Eintragungen wurde jedoch nicht erreicht.

Eine Volksinitiative des Mieterbundes zur Schaffung sozialer und rechtlicher Voraussetzungen bei der Überleitung in das Vergleichsmietensystem erreichte das Quorum ohne nennenswerte Probleme, der Landtag entsprach dem Anliegen, indem die Landesregierung auf eine entsprechende Einwirkungsweise im Bundesrat durch Landtagsbeschluss festgelegt wurde.

Ein Gesetz zur Förderung von Musikschulen im Land Brandenburg wurde durch eine Volksinitiative 1996 unterstützt, ein ausformulierter Gesetzentwurf wurde vorgelegt. Auch in diesem Falle wurde das Quorum erreicht, das Parlament lehnte das Anliegen jedoch ab. Der Antrag auf Volksbegehren wurde nach zwei Monaten zurückgezogen. Erreicht wurde die Fortschreibung der Förderung der Musikschulen durch das Land fuir den Zeitraum der zweiten Wahlperiode, d. h. bis 1999, auf dem Niveau von 1996.

Eine Volksinitiative betraf die Abwasserproblematik, sie agierte „für sozialverträgliche Wasser- und $\mathrm{Ab}$ wasserpreise“. Die Initiative brachte 21.133 mängelfreie Unterschriften. Die Unterzeichner wollten, dass sich das Parlament speziell in der zweiten Wahlperiode wiederholt mit der juristischen und wirtschaftlichen Situation und Entwicklung im Abwasserbereich befasste. Es kam zu Protestmärschen zum Deutschen Bundestag nach Berlin, zur Europäischen Kommission nach Brüssel und zu einem Hungerstreik im Landtagswahlkampf 1999. Im Sommer 2000 gab es in der Gemeinde Brieselang einen Hungerstreik gegen den gegenüber einer dezentralen Lösung fünffach teureren Zwangsanschluss. [3] 
Eine Volksinitiative „für ein Gesetz zur Förderung der Jugendarbeit und Jugendsozialarbeit" erreichte nicht die Mindestanzahl mängelfreier Unterschriften.

Bemerkenswert erscheint indes, dass die im Volksgesetzgebungsgesetz vorgesehene mögliche Herabsetzung der Altersgrenze zur Beteiligung an der Volksinitiative von 18 auf 16 Jahre auch durch Beschluss im Hauptausschuss ermöglicht wurde, wobei alle im Parlament vertretenen Parteien (SPD, CDU, PDS) diesem Schritt zustimmten.

Im April 1997 wurde die Volksinitiative gegen den Transrapid vorstellig, das Quorum wurde erreicht, der Landtag lehnte das Anliegen indes ab. Im darauf folgenden Volksbegehren wurden 69.570 gültige Eintragungen erreicht, also erneut weniger als die erforderlichen 80.000 Eintragungen. Während beim Volksbegehren gegen den Havelausbau eine deutliche Konzentration der Eintragungen auf die betroffenen kreisfreien Städte Potsdam und Brandenburg sowie auf den Landkreis Potsdam-Mittelmark auszumachen war [4], waren beim Volksbegehren gegen den Transrapid deutlich mehr Eintragungen aus den nicht direkt betroffenen, d. h. also nicht unmittelbar an der geplanten Strecke liegenden Kreisen festzustellen.

Im Sommer 1998 brachte eine Volksinitiative gegen den Ausbau des Flughafens Berlin-Schönefeld zum Großflughafen Berlin-Brandenburg-International 24.000 Unterschriften ein. Der Landtag lehnte diese Vorlage ab und beauftragte die Landesregierung, sich im Bundesrat für Verbesserungen im Lärm- und Gesundheitsschutz einzusetzen.

Vor Ablauf der zweiten Wahlperiode wurde der Landtag mit einer Volksinitiative von etwa 200.000 Unterschriften „gegen die zunehmende Benachteiligung der ostdeutschen Bevölkerung in der medizinischen Betreuung und Versorgung" konfrontiert. Der Landeswahlleiter fand von 67.700 untersuchten Unterschriften 47.977 ohne Mängel, d. h. das Quorum wurde erfuillt. Zum Gegenstand, dem Risikostrukturausgleich für die Finanzausstattung des Gesundheitswesens im Osten Deutschlands, wurde die Landesregierung zur Fortsetzung ihres Engagements aufgefordert.

\section{Dritte Wahlperiode, ab 1999}

Mit dem Programm der seit 1999 neuen Regierung, deren Zustandekommen - durch Wahrnehmung des Wahlrechtes - selbst als Ausdruck des Wählerwillens zu verstehen ist, gibt es gegenüber den vorigen Wahlperioden bemerkenswerte Veränderungen festzuhalten: Erstes Bezugsdokument ist hierfür die Koalitionsvereinbarung der die Regierung tragenden Parteien bzw. ihrer Fraktionen. Prägend sind dafür die beabsichtigten Veränderungen in den Bereichen der Kinderbetreuung, der Forstreform und nicht zuletzt der Gemeindegebietsreform.

Im Kita-Bereich wurden Reform- und Sparabsichten darauf orientiert, einen bis dahin ohne Bedingungen geltenden, d. h. nicht konditionierten Rechtsanspruch auf einen Kita-Platz künftig durch einen konditionierten
Rechtsanspruch zu ersetzen: Wenn etwa für die Arbeitsaufnahme der Mutter eine Kinderbetreuung erforderlich wird, so muss die Betreuung auch realisiert werden. Mit dieser Veränderung beim Rechtsanspruch auf einen KitaPlatz werden Verhältnisse geschaffen, die näher an den Bedingungen anderer Bundesländer liegen. Kita-Erzieherinnen und Lehrer protestierten gegen diese politischen Absichten. Es wurden zwar 152.000 Unterschriften [5] gegen das Vorhaben gesammelt, das Landesparlament stimmte jedoch den von der Regierung vorgesehenen Änderungen zu.

Eine weitere Strukturveränderung im Land wurde durch die beabsichtigte Forstreform in Angriff genommen. Etwa 10 Jahre nach Gründung des Bundeslandes wurde eingeräumt, dass die staatliche Forstverwaltung als Erbe aus der DDR-Zeit mit immer noch gut 3.000 Mitarbeitern stark überbesetzt sei und deshalb bis 2010 halbiert werden solle [6]. So soll die staatliche Forstverwaltung ab 2002 in eine Anstalt öffentlichen Rechts umgewandelt werden, die eigene wirtschaftliche Anstrengungen zu unternehmen hat. Dagegen werden Überlegungen, ein Eintrittsgeld in den Wald zu verlangen, das vom Koalitionspartner mit 10 DM „bemessen“ wurde, seitens des Ministers verworfen. Die Zahl der Waldarbeiter soll um 600 reduziert werden. In Zusammenhang mit dieser beabsichtigten Forstreform wurden 140.000 Unterschriften gegen die Reform an den Landtag übergeben [7|. Die Initiatoren kündigten an, sich der Reformabsicht mit allen Mitteln zu widersetzen, wobei sie Verfassungsklage und Volksinitiative nannten.

Eine dritte das gesamte Land Brandenburg betreffende Reform stellt die Gemeindegebietsreform dar, die im Koalitionsvertrag aufgenommen ist. Nach der Bildung des Landes 1990 und der Kreisgebietsreform 1993, bei der die bis dahin 38 Landkreise und 6 kreisfreien Städte in 14 Landkreise und 4 kreisfreie Städte umgewandelt wurden, setzte der 2. Landtag eine Enquete-Kommission zur Gemeindegebietsreform ein, die im April 1999 einen Bericht vorlegte. Diesem entnimmt man die Grundtendenz, kleine Gemeinden in größeren Gemeinden, sogenannten Amtsgemeinden, zusammenzufassen. Dabei orientierte man sich an Rheinland-Pfalz, wobei ein vollständiger Vergleich den Hinweis gebietet, dass die dortigen kleinen Ortsgemeinden gegenüber der übergeordneten Verbandsgemeinde (denen in Brandenburg die Amtsgemeinde entsprechen soll) ihre Kompetenzen umfangreich wahrnehmen, wohl auch dank des bürgerschaftlichen Engagements. Der Bericht der Enquetekommission Gemeindegebietsreform, deren Zusammensetzung dem Stärkeverhältnis im zweiten Brandenburger Landtag entsprach, ist mittlerweile selbst Gegenstand verwaltungswissenschaftlicher Untersuchungen [8]. Da in der zweiten Wahlperiode die Reform selbst nicht in Angriff genommen wurde, bleibt deren Thematisierung für die dritte Wahlperiode. Der Regierungswechsel von 1999 führte dabei zu durchaus zu differenzierenden Positionsveränderungen bei den Koalitionspartnern. Mit der Vorlage von Leitlinien des Innenministers und deren Billigung durch das Kabinett Anfang Juli 2000 wurde ein Zeitplan skizziert, nach dem einer „Frei- 
willigkeitsphase" von 18 Monaten gesetzlich vorgeschriebene Gemeindestrukturveränderungen folgen sollten mit dem Ziel, im Land Brandenburg größere Gemeinden zu schaffen. Durch einen Bürgermeister, der angibt insbesondere die kleinen Gemeinden zu vertreten, wurde diesem Vorgehen entgegengetreten und 9.000 Unterschriften gegen das Vorhaben gesammelt. Zwar ist das Quorum für eine Volksinitiative damit ersichtlich nicht erreicht, jedoch hat sich in diesem Kontext ein „Gemeindetag Brandenburg“ konstituiert, der die Interessen der kleinen Gemeinden vertreten will.

\section{Nutzt die Opposition diese Reformen zur Wahrnehmung ihrer Integrationsfunktion?}

In diesen drei wesentlichen, die Struktur des Landes (und seines Haushaltes) betreffenden Reformvorhaben sind neben der Regierungskoalition die parlamentarische Opposition und/oder die nichtparlamentarischen Kräfte in ihrer Willensbildung und Willensartikulation zu erfassen:

Kita-Reform und Forstreform werden von der PDS abgelehnt, die Gemeindereform wird in Grundziigen mitgetragen. Der Vollständigkeit halber wird vermerkt, dass die DVU alle Reformen ablehnt.

Gegenuiber der nichtparlamentarischen Willensbildung gibt es somit grundsätzlich die Möglichkeit, dass die Opposition ihre Integrationsfunktion wahrnimmt, indem sie diese Positionen aufgreift bzw. vertritt.

Nuitzt das der Opposition?

Freilich gehört es auch grundsätzlich zur politischen Praxis in den östlichen Bundesländern, dass durch die Wahrnehmung der Integrationsfunktion der Oppositionsparteien nicht die Mitgliedschaft in diesen Parteien wächst, allenfalls entstehen Wahlpräferenzen, mehr noch resultieren daraus wohl Enttäuschung bei den Bürgern und Wahlenthaltung.

\section{Kann man also von einem "Missbrauch der Volksgesetzgebung" ernsthaft sprechen?}

Vom Verfahren und von der Tragweite her sind Volksinitiative und Volksbegehren zum Landesmusikschulgesetz von diesen Reformvorhaben zu unterscheiden, nicht dagegen vom Resultat her: Eine Volksinitiative fuir ein Landesmusikschulgesetz [9] erreichte 1996 und erneut $1999[10]$ sicher das in Brandenburg erforderliche Quorum von 20.000 Unterschriften. Erstmals wurde wie oben ausgeführt - dabei dem Parlament ein Gesetzentwurf vorgelegt, was bei anderen Volksinitiativen nicht der Fall war [11]. Nachdem der Landtag auch dieses Anliegen ablehnte, wurde das Volksbegehren, welches vom März bis Juli 2000 stattfand, beantragt. Im Gegensatz zu den Volksbegehren aus der zweiten Wahlperiode, die im Falle des Transrapids und des Havelausbaus Teile des Landes, nicht dagegen das ganze Land betrafen, schienen mit der beabsichtigten Landesgesetzregelung deutlich bessere Voraussetzungen für ein erfolgreiches Volksbegehren gegeben. Der massive Widerstand der Landkreise gegen eine gesetzliche Festschrei- bung führte jedoch zu einer Paralysierung der Akteure in zahlreichen Musikschuleinrichtungen, sodass eine flächendeckend hinreichende [12] Unterschriftenerfassung nicht erfolgte. Wie in den Volksbegehren zuvor erwies sich auch in diesem Fall der Aufwand, „im Amt seine Unterschrift zu leisten“, als erheblich. (Bei der Volksinitiative geniigte es, die Unterschrift ,auf der Strasse zu leisten“.) Hinzu kam, dass die Landesregierung zeitgleich im Sommer 2000 einen Gesetzentwurf vorlegte mit dem Anspruch, das Anliegen in wichtigen Teilen aufgenommen zu haben.

\section{Zusammenfassung}

In jeder Wahlperiode ist ein anderer Charakter der Volksgesetzgebungsschritte festzustellen: In der ersten Wahlperiode betrafen Volksgesetzgebungsschritte im wesentlichen die Kreisgebietsreform, in der zweiten Wahlperiode war ein Spektrum von verschiedenen Themen Gegenstand von Volksinitiativen. Bis auf das Anliegen des Mieterverbandes hat sich das Landesparlament keines der Themen der (außerparlamentarisch initiierten) Volksgesetzgebung zueigen gemacht. In der ersten Hälfte der dritten Wahlperiode erfolgte die nichtparlamentarische Willensbildung und Willensartikulation gegen wesentliche Reformvorhaben einer großen Koalition. Die Gegner dieser in Brandenburg in diesem Sinne eher nachzuarbeitenden Reformen agieren nicht einmal vorrangig durch Inanspruchnahme der gesetzlich vorgeschriebenen Instrumentarien der Volksgesetzgebung, die in Brandenburg besonders häufig Anwendung finden: Man erstellt Unterschriftensammlungen und gibt diese als Petition an das Landesparlament oder das zuständige Regierungsmitglied. Dem Anliegen der Antragsteller dürfte auf diesem Wege substanziell jedoch ebenso wenig entsprochen werden.

Die parlamentarische Opposition kann durch die Artikulation dieser nichtparlamentarischen Positionen nicht unmittelbar profitieren, neben dem Wandel von Wahlpräferenzen gewinnt die Partei der Nichtwähler an Zuspruch.

Falls sich also bei der Verabschiedung der Landesverfassung Brandenburg 1992 mit ihren im Bundesmaßstab vergleichsweise geringen Quoren zur Volksgesetzgebung die Erwartung dargestellt haben mag, dass sich durch Inanspruchnahme der Volksgesetzgebung „ein Sonderweg“" oder „der Weg in eine andere Republik" abzeichnen würde, so bleibt festzuhalten, dass dies sicher nicht der Fall ist. Die Erfolgsquote von Volksgesetzgebungsschritten ist gering. Ein Sonderweg Brandenburgs, gar in eine andere Republik, ist also nicht auszumachen.

\section{Anmerkungen}

[1] So der ehemalige Oppositionsführer der CDU/CSU im Deutschen Bundestag, Rainer Barzel, in der Verfassungsdebatte.

[2] Vgl. Klages/Paulus: Direkte Demokratie in Deutschland, Schüren, 1996 
[3] So beispielsweise im ORB-Hörfunk am 06.08.2000 und in den Potsdamer Neueste Nachrichten (PNN) am 08.08.2000 berichtet.

[4] S. Auswertung durch das LDS Brandenburg.

[5] So die am 13.07.2000 vorgelegte (18.) Volksinitiative in Brandenburg.

[6] Märkische Allgemeine Zeitung (MAZ), 03.05.2000

[7] PNN, 03.05.2000

[8] S. Gruinewald apf August 1999, Br 57.

[9] Die mögliche Herabsetzung des Alters zur Unterschriftsleistung bei der Volksinitiative auf 16 Jahre gemäß Volkabstimmungsgesetz erfolgte in beiden Fällen.

[10] Am 19.07.1999 wurden 61.022 Unterschriften vorgelegt, von 29.799 geprüften erwiesen sich 22.525 als mängelfrei.

[11] In anderen Bundesländern ist die Vorlage eines Gesetzentwurfes ein zu erfüllendes Kriterium, was für die Vergleichbarkeit von Volksgesetzgebungsschritten zwischen Bundesländern nicht unberiicksichtigt bleiben soll.

|12] Auswertung des Landeswahlleiters vom 10.08.2000.

\section{Literatur}

Der Landeswahlleiter: Mitteilungen zu Volksinitiativen und Volksbegehren

Hauptausschuss des Landtages: Protokolle und Beschlussempfehlungen

Klages, Andreas; Paulus, Petra: Direkte Demokratie in Deutschland, Schüren, 1996

Koalitionsvereinbarung Brandenburg vom 12.10.1999

Vette, Markus: Zur Struktur und Übernahme politischer Verantwortung in der repräsentativen parlamentarischen Demokratie - Erfahrungen und Schlussfolgerungen aus der Arbeit des ersten Brandenburger Landtages; uni-press Bd. 86; LIT Münster, 1996

\section{Autor}

Dr. rer. nat. Dr. phil. Markus Vette

Technische Fachhochschule Wildau

Fachbereich Wirtschaft/Nerwaltung und Recht

Tel. (0 33 75) 508-570

E-Mail: markus@vette-net.de 